\title{
Inhibition of Melanosis in Whiteleg Shrimp (Litopenaeus vannamei) during Refrigerated Storage Using Extracts of Different Avocado (Persea americana Mill.) By-Products
}

\author{
Dao Thi Anh Phan ${ }^{1}$, Trung Huu Bui ${ }^{1}$, Tram Quynh Thi Doan ${ }^{1}$, Nguyen Van Nguyen², and Trieu Hai Ly ${ }^{3}$ \\ ${ }^{1}$ Faculty of Chemical and Food Technology, HCMC University of Technology and Education, Ho Chi Minh 70000, Viet Nam \\ ${ }^{2}$ Research Center for Aquafeed Nutrition and Fishery Post-Harvest Technology (APOTEC), Ho Chi Minh 70000, Viet Nam \\ ${ }^{3}$ Research Center of Ginseng and Medicinal Materials, National Institute of Medicinal Materials, Ho Chi Minh 70000, Viet Nam
}

\begin{abstract}
Melanosis in shrimp usually leads to reduction in its shelf life and quality, which causes a significant loss in economic value of shrimp products. This study reports potential applications of nine ethanolic extracts of by-products, i.e., peel and/or seed from three Vietnamese avocado varieties as effective inhibitors of melanosis in whiteleg shrimp. Six out of nine shrimp samples treated with the prepared extracts $(0.025 \%, \mathrm{w} / \mathrm{v})$ reduced melanosis and lipid oxidation more significantly as compared to those treated with sodium metabisulfite (SMS, $1.25 \%$, w/v) and control groups (treated with water) during 8 -day storage at $4^{\circ} \mathrm{C}(P<0.05)$. These six extracts had mean gray values ranging from $47.0 \pm 0.7$ to $57.3 \pm$ $0.4 \%$ were lower than those treated with SMS (mean gray of $39.8 \pm 0.4 \%$ ). The inhibition of melanosis and lipid oxidation in shrimp for these extracts could be attributed to their high content of polyphenols [total phenolic content (TPC) from $44.5 \pm 1.1$ to $144.7 \pm 1.9 \mathrm{mg}$ gallic acid equivalents/g dried weight] and strong antioxidant activities [including 2,2-diphenyl-1-picrylhydrazyl (DPPH) radical scavenging, ferric reducing antioxidant power (FRAP), and tyrosinase enzyme inhibition]. Pearson statistical analysis showed strong correlation for melanosis inhibition to TPC and DPPH scavenging ( $r>$ $0.80)$ followed by tyrosinase inhibition and FRAP $(r>0.50)$. The findings obtained from this study suggest potential utilization of avocado by-product extracts as safe and cheap natural alternatives to traditional sulfites for anti-melanosis and shelf life extension of whiteleg shrimp.
\end{abstract}

Keywords: antioxidant, avocado by-products, melanosis, tyrosinase, whiteleg shrimp

\section{INTRODUCTION}

Whiteleg shrimp (Litopenaeus vannamei) is one of the most widely cultured and consumed shrimp species because of its delicacy, accounting for $80 \%$ of the global shrimp production (Jescovitch et al., 2018). However, this highvalue shrimp has a limited shelf life, in which melanosis (or black spot) formation during postmortem storage is problematic (Gonçalves and Oliveira, 2016), and causes substantial loss in the nutritional and economic value of these shrimp products.

Melanosis in shrimp and other crustaceans is developed by the oxidation of phenol substrates (such as tyrosine in shrimp) to quinones catalyzed by polyphenolase, which generates dark pigments of high molecular compounds (Sae-leaw and Benjakul, 2019). From a commercial perspective, some methods have been used to prevent mela- nosis development in shrimp during storage, such as treatments with sulfite and phosphate compounds (Martínez-Álvarez et al., 2008; Galvão et al., 2017). However, the potential risks of using those chemical additives, i.e., nausea, abdominal pain, vomiting, and choking have raised awareness among customers and regulators. Consequently, researchers and scientists worldwide have recently paid considerable attention to natural, safe, and effective additives (Djeridane et al., 2006; Encarnacion et al., 2012). Natural antioxidant substances have been widely studied as substitutes to chemical additives to prevent melanosis. Natural additives, such as tocopherols, flavonoids, cinnamic acids, and coumarins were reported to exhibit potent antioxidant, antimicrobial, and polyphenoloxidase (PPO) inhibitory activities and thus, they could prevent the melanosis process (Gonçalves and de Oliveira, 2016). Plant extracts containing polyphenol- 
ic compounds demonstrated a significant retardation of melanosis formation in crustaceans, which was associated with tyrosinase inhibitory activity (Sae-leaw and Benjakul, 2019). Nirmal and Benjakul (2012) reported that the ethanolic extract of green tea containing a high amount of catechin and derivatives could significantly decrease the lipid oxidation and melanosis formation in whiteleg shrimp during cold storage. Encarnacion et al. (2012) prepared an extract of edible mushroom (Flammulina velutipes) containing rich ergothioneine, which showed positive effects on melanosis prevention in shrimps Penaeus monodon and Litopenaeus vannamei. Chamuang leaf extract and lead (Leucaena leucocephala) seed extract that were rich in phenolic compounds effectively inhibited melanosis in whiteleg shrimp (Nirmal and Benjakul, 2011; Shiekh et al., 2019).

Recently, studies on utilizing agricultural by-products for food additives and preservation have been extensively investigated due to economic and environmental benefits (Gómez et al., 2014; Saavedra et al., 2017). Among various by-products, avocado seed and peel contained great amounts of phenolic compounds, including flavanol monomers, proanthocyanidins, hydroxycinnamic acids, and flavonol glycosides (Kosińska et al., 2012). This would attribute to a high potential of antioxidant activities of those by-products. Rodríguez-Carpena et al. (2011) showed that raw porcine patties treated with the extracts of avocado peel and seed could effectively inhibit lipid oxidation and color deterioration during chilled storage. Several studies also reported the applications of avocado byproducts as potential sources of natural antioxidants or preservatives (Gómez et al., 2014: Saavedra et al., 2017; Gashahun and Solomon, 2018).

It is worth mentioning that avocado peel and seed are discarded as wastes, accounting for about $20 \%$ (w/w) of the fruit (Wang et al., 2010). Avocados are widely grown in Viet Nam, which has been considered as one of the seven important fruits crops in the country. Consequently, a large quantity of avocado by-products is discarded from fruit processing annually. Although the phenolic compounds, such as catechin, quercetin, and their derivatives from avocado by-products could exhibit a potential in preventing melanosis on crustaceans or shrimps (Nirmal and Benjakul, 2012; Qian et al., 2018; Tremocoldi et al., 2018; Rosero et al., 2019), to the best of our knowledge, there has been no report on utilizing avocado byproducts as potential natural additives to prevent melanosis and/or extend the shelf life of whiteleg shrimp.

In this study, the roles of some popular avocado byproducts on the retardation of melanosis and lipid oxidation in whiteleg shrimp during cold storage were evaluated and discussed. The study aimed to obtain three main objectives: (i) preparation of nine ethanolic extracts from by-products (seeds and peels) of three avocado va- rieties from Viet Nam, and determination of their total phenolic content (TPC) and antioxidant activities; (ii) evaluation of the capability of these prepared extracts in preventing melanosis formation and lipid oxidation in whiteleg shrimp during 8 days storage at $4^{\circ} \mathrm{C}$; and (iii) discussion on the relationship between the inhibition on melanosis of these prepared extracts and their TPC values and antioxidant activities. The utilization of these byproducts as food additives could not only reduce the negative impact on the environment but also expand the range of avocado value-added products.

\section{MATERIALS AND METHODS}

\section{Materials and chemicals}

Folin-Ciocalteu reagent, iron (III) chloride hexahydrate $\left(\mathrm{FeCl}_{3} \cdot 6 \mathrm{H}_{2} \mathrm{O}\right)$, malondialdehyde (MDA), sodium metabisulfite (SMS), potassium hexacyanoferrate $\left[\mathrm{K}_{3} \mathrm{Fe}(\mathrm{CN})_{6}\right]$, thiobarbituric acid (TBA), trichloracetic acid (TCA), gallic acid, kojic acid, 2,2-diphenyl-1-picrylhydrazyl (DPPH), and tyrosinase were purchased from Sigma-Aldrich Co. (St. Louis, MO, USA). All other chemicals were of analytical grade.

By-products (peels and seeds) from three popular varieties of avocado, namely 'Nuoc', 'Sap', and '034' were supplied from several local markets in Ho Chi Minh City, Viet Nam. The samples were then dried to the moisture content of about $10 \%$ [using a gravimetric method followed by the European Brewery Convention (2000)]. The dried samples were subsequently ground in a blender into a homogenous fine pulp that was then extracted with ethanol.

Alive whiteleg shrimp with the size of 30 40 shrimps/ $\mathrm{kg}$ were purchased from Tan Binh market (Ho Chi Minh City, Viet Nam). The shrimps were immediately transported to the laboratory for the experiments.

\section{Preparation of extracts}

An extract of each avocado by-product was prepared using a maceration technique (Azwanida, 2015). Dried powder $(30 \mathrm{~g})$ was soaked in $100 \mathrm{~mL}$ of $70 \%$ ethanol $(\mathrm{v} / \mathrm{v})$ at $32^{\circ} \mathrm{C}$ for $24 \mathrm{~h}$. The mixture was filtered to collect the extract solution, and the remaining residue was re-extracted with ethanol twice using the same procedure. Then, the mixture of three collected extracts was concentrated using a rotary evaporator to produce a crude extract. Table 1 presents the extraction yield (E\%) of the nine prepared avocado by-product extracts. It includes three seed extracts and three peel extracts prepared from each variety ['Sap', '034', and 'Nuoc': No. (1) to (6)], and three mixed extracts [No. (7) to (9)] consisting of peel mixture, seed mixture, and peel-seed mixture from 3 varieties mixed in equal weights. Note, the extraction yield 
Table 1. The extraction yield of nine extracts prepared from by-products of three avocado varieties: 'Sap', '034', 'Nuoc'

\begin{tabular}{cclc}
\hline No. & $\begin{array}{c}\text { Sample } \\
\text { name }\end{array}$ & \multicolumn{1}{c}{ Formulation/mixture } & $\begin{array}{c}\text { Extraction } \\
\text { yield (\%) }\end{array}$ \\
\hline 1 & SS & Sap seed & 12.5 \\
2 & SP & Sap peel & 10.0 \\
3 & OS & O34 seed & 10.1 \\
4 & OP & O34 peel & 7.6 \\
5 & NS & Nuoc seed & 18.1 \\
6 & NP & Nuoc peel & 7.8 \\
7 & MS & Sap seed + 034 seed + Nuoc seed & 13.5 \\
8 & MP & Sap peel + 034 peel + Nuoc peel & 7.6 \\
9 & MSP & Mixture of all seeds and peels & 9.8 \\
\hline
\end{tabular}

(E\%) can be calculated as in Eq. (1):

$$
\text { Yield }(\%)=\frac{m_{\text {extract }}}{m_{\text {powder }}} \times 100
$$

where $m_{\text {extract }}$ is the weight $(\mathrm{g})$ of the dried extract residue obtained after solvent removal, and $\mathrm{m}_{\text {powder }}$ is the weight $(\mathrm{g})$ of plant powder.

\section{Determination of TPC}

The TPC of the prepared extracts was determined using the colorimetric Folin-Ciocalteu method (Jung et al., 2008). The calibration curve was constructed using gallic acid as standard at a concentration range of 0 to $20 \mathrm{mg} / \mathrm{L}$, showing a linear equation of $y=0.1027 x+0.0365\left(\mathrm{R}^{2}=\right.$ 0.9979). The TPC value was expressed as mg gallic acid equivalents (GAE) per gram dried weight (g DW). Each experiment was performed in triplicate.

\section{Antioxidant activities}

DPPH assay: The scavenging activity of DPPH radical was performed using a method documented by Molyneux (2004). Each sample $(1.5 \mathrm{~mL})$ at four concentrations of $10,25,50$, and $100 \mu \mathrm{g} / \mathrm{L}$ (in $90 \%$ ethanol) was mixed with $1.5 \mathrm{~mL}$ of $0.1 \mathrm{mM}$ DPPH solution and the mixture was then incubated for 30 min under dark. The absorbance of the obtained mixture and blank samples (without extract) was measured at $519 \mathrm{~nm}$. The inhibitory percentage of DPPH from the tested samples was calculated as in Eq. (2):

$$
\mathrm{I}(\%)=\left(1-\frac{\mathrm{B}}{\mathrm{A}}\right) \times 100
$$

where $\mathrm{A}$ is the absorbance of the test sample, and B is the absorbance of the blank sample.

The half-maximal inhibitory concentration $\left(\mathrm{IC}_{50}\right)$ was calculated from the mean values of three measurements at the used concentration range. Gallic acid was used as a positive control with the $\mathrm{IC}_{50}$ of $5.62 \mu \mathrm{g} / \mathrm{mL}$.
Ferric reducing antioxidant power assay: Ferric reducing antioxidant power (FRAP) assay was conducted according to the method reported by Yen and Chen (1995). Different amounts of each extract $(0.1,0.5$, and $1.0 \mathrm{mg}$ ) were mixed with $1 \mathrm{~mL}$ of $2 \mathrm{M}$ phosphate buffer ( $\mathrm{pH} \mathrm{6.6)}$ and $1 \mathrm{~mL}$ of $1 \% \mathrm{~K}_{3} \mathrm{Fe}(\mathrm{CN})_{6}$. After incubating for $20 \mathrm{~min}, 1$ $\mathrm{mL}$ of $10 \%$ TCA was added, blended, and centrifuged at 4,000 rpm for $10 \mathrm{~min}$ to collect the supernatant. Subsequently, $1 \mathrm{~mL}$ of the supernatant was diluted with $1 \mathrm{~mL}$ of deionized water and then mixed with $0.2 \mathrm{~mL}$ of $\mathrm{FeCl}_{3}$ $(0.1 \%)$. The absorbance was measured at $700 \mathrm{~nm}$. The reducing capacity was calculated based on the increase in absorbance. Vitamin $\mathrm{C}$ was used as a positive control at various concentrations $(0.1,0.5$, and $1.0 \mu \mathrm{M})$.

Tyrosinase inhibitory assay: Tyrosinase inhibitory activity was performed following the procedure established by Nirmal and Benjakul (2011), with slight modifications using a $96-$ well reader. Each $40 \mu \mathrm{L}$ of the extract $(100 \mu \mathrm{g} /$ $\mathrm{mL}$ ) mixed with $20 \mu \mathrm{L}$ of tyrosinase $(100 \mathrm{U} / \mathrm{mL})$ was incubated at room temperature for $30 \mathrm{~min}$. The reaction was initiated with the addition of $140 \mu \mathrm{L}$ of $0.714 \mathrm{mM}$ L-3,4-dihydroxyphenylalanine (L-DOPA) at $45^{\circ} \mathrm{C}$ for 5 $\min$. The absorbance of the mixture was then measured at $475 \mathrm{~nm}$. The tyrosinase inhibitory percentage and $\mathrm{IC}_{50}$ values were calculated similarly to those of the DPPH assay. Kojic acid was used as a positive control with the $\mathrm{IC}_{50}$ value of $42.80 \mu \mathrm{g} / \mathrm{mL}$.

\section{Evaluation of melanosis formation and lipid peroxidation inhibition in shrimp}

Shrimp treatment: Shrimp were divided into 11 equal parts. Each part was immersed in either each of the extract solutions $(0.025 \%, \mathrm{w} / \mathrm{v})$, or the SMS solution $(1.25 \%, \mathrm{w} /$ $\mathrm{v}$ ), or deionized water (as the control), in the ratio of 1:2 (shrimp/solution, w/v) at $4^{\circ} \mathrm{C}$ for $15 \mathrm{~min}$. Subsequently, the treated shrimp were placed in polypropylene boxes and stored at $4^{\circ} \mathrm{C}$ for eight days. Note that the control samples, known as blank samples, were performed by using deionized water as a replacement for the extract solutions.

Melanosis evaluation: Visual images of the shrimp samples during storage were taken daily. The changes in color of the samples were analyzed using ImageJ software (version 1.52a, National Institutes of Health, Bethesda, MD, USA). Relative changes in the gray value of the carapace were calculated as in Eq. (3) (Encarnacion et al., 2012; Sae-leaw and Benjakul, 2019). The decrease in the \% relative change indicates an increase of melanosis formation in shrimp during storage. Three shrimps were used for one treatment:

$$
\% \text { Relative change }=100-\frac{C \times 100}{D}
$$


where $\mathrm{C}$ is the actual gray value of the shrimp sample, and $\mathrm{D}$ is the average gray value of the shrimp on the first day.

Lipid peroxidation inhibition assay: The peroxidation products (TBA reactive substances, TBARS) produced during shrimp preservation were analyzed using the procedure established by Benjakul and Bauer (2001). Ground shrimp $(10 \mathrm{~g})$ were thoroughly mixed with $10 \mathrm{~mL}$ of $7.5 \% \mathrm{w} / \mathrm{v}$ TCA and filtered to collect the filtrate. Subsequently, the filtrate was mixed with $0.02 \mathrm{M}$ TBA solution (at the same volume ratio) and incubated at $100^{\circ} \mathrm{C}$ for $15 \mathrm{~min}$. The mixture was then cooled to about $32^{\circ} \mathrm{C}$, and the absorbance was measured at $532 \mathrm{~nm}$. The calibration curve was constructed using MDA standard solutions at concentrations from 0.01 to $0.05 \mu \mathrm{M}$. The obtained linear equation was $y=0.0441 x+0.0148\left(\mathrm{R}^{2}=0.9993\right)$. The TBARS values were expressed as $\mathrm{mg} \mathrm{MAD} / \mathrm{kg}$ shrimp.

\section{Analysis method}

Instrument analysis: A Hitachi UH-530 ultraviolet/visible spectrophotometer (Hitachi, Ltd., Tokyo, Japan) was used to measure the absorbance at a specific wavelength conducted in the experiments for TPC, antioxidant assays, and lipid peroxidation assay. Photographs of the shrimps during 8 days storage were taken using a Canon Eos M10 Kit Ef-M15-45 (Canon Inc., Tokyo, Japan). An ImageJ software (version 1.52a, National Institutes of Health) was used to analyze the color changes for melanosis determination.

Statistical analysis: Experimental data were expressed as mean \pm standard deviation (SD) and analyzed by analysis of variance (ANOVA) with Tukey's test $(P<0.05)$. All measurements were replicated three times. Statistical calculations were carried out using a SPSS package (SPSS 20 for Windows Evaluation Version, IBM Corporation, Armonk, NY, USA).

\section{RESULTS AND DISCUSSION}

\section{Total phenolic content}

Fig. 1 shows the TPC of the prepared extracts. All extracts had high TPC with values ranging between $44.5 \pm$ 1.1 ['034' seed (OS)] and 144.7 $\pm 1.9 \mathrm{mg}$ GAE/g DW ['Nuoc' peel $(\mathrm{NP})](P<0.05)$. For each type of avocado by-product, the extract from the peel exhibited higher TPC than that from the seed ['Sap' peel (SP) $>$ 'Sap' seed (SS), '034' peel (OP) > OS, and NP> 'Nuoc' peel (NS), respectively]. Similar results were reported in a previous study on TPC in Hass and Fuerte avocado (Tremocoldi et al., 2018). Specifically, the extracts of 'Nuoc' variety exhibited the highest TPC values (114.7 \pm 3.5 and 144.7 $\pm 1.9 \mathrm{mg}$ GAE/g DW for NS and NP, respectively), followed by 'Sap' and '034' avocado by-product extracts

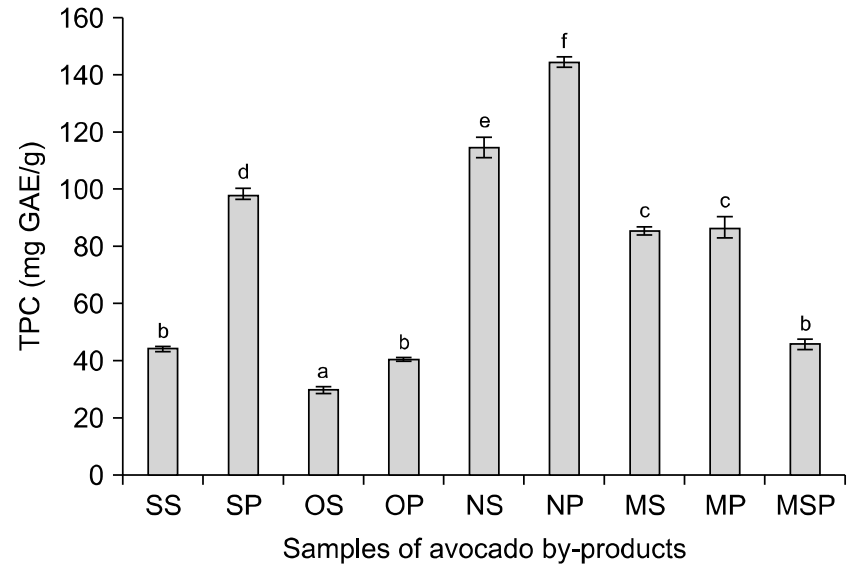

Fig. 1. Total phenolic content (TPC) of 9 avocado by-product extracts [Sap seed (SS), Sap peel (SP), 034 seed (OS), 034 peel $(\mathrm{OP})$, Nuoc seed (NS), Nuoc peel (NP), seed mixture (MS), peel mixture (MP), and peel and seed mixture (MSP)]. Values represent the mean \pm standard deviation $(n=3)$. Different letters $(a-f)$ indicate significant differences $(P<0.05)$.

$(P<0.05)$. It is clearly seen that TPC values for the three mixed extracts [mixture of all seeds (MS), mixture of all peels (MP), and mixture of all seeds and peels (MSP)] were found in the order of MSP $(45.8 \pm 2.0 \mathrm{mg} / \mathrm{g} \mathrm{DW})<$ MS $(85.52 \pm 1.4 \mathrm{mg} / \mathrm{g} \mathrm{DW})<$ MP $(86.6 \pm 3.8 \mathrm{mg} / \mathrm{g} \mathrm{DW})$ $(P<0.05)$. The results of TPC in this study were comparable or even higher than those reported in a previous study for other types of avocado by-product, e.g., Hass peel $(63.5 \pm 7.2 \mathrm{mg} / \mathrm{g}$ DW), Hass seed $(57.3 \pm 2.7 \mathrm{mg} / \mathrm{g}$ DW), Fuerte peel $(120.3 \pm 7.8 \mathrm{mg} / \mathrm{g} \mathrm{DW})$, and Fuerte seed $(59.2 \pm 6.9 \mathrm{mg} / \mathrm{g} \mathrm{DW})$ (Tremocoldi et al., 2018). Githinji et al. (2013) showed the TPC of Fuerte seed extract was only $18.5 \pm 2.8 \mathrm{mg} / \mathrm{g}$ DW. The TPC of avocado seed and peel $[29.9 \pm 1.1(\mathrm{OS})$ and $144.7 \pm 1.9$ (NP) $\mathrm{mg}$ GAE/g DW respectively] in this study was greater than that reported for blueberry $(9.44 \pm 0.22 \mathrm{mg} \mathrm{GAE} / \mathrm{g} \mathrm{DW})$, blackberry (5.58 $\pm 0.18 \mathrm{mg} \mathrm{GAE} / \mathrm{g} \mathrm{DW})$, and strawberry $(2.72 \pm 0.18 \mathrm{mg} \mathrm{GAE} / \mathrm{g} \mathrm{DW})$, all of which have been known for their high antioxidant capacity (Huang et al., 2012). A variety of phenolic compounds has been reported in avocado seed and peel by-products such as, flavanol monomers, proanthocyanidins, hydroxycinnamic acids, and flavonol glycosides, 3-O-caffeoylquinic acid, 3-O-p-coumaroylquinic acid, and procyanidin A trimers (Kosińska et al., 2012). Tremocoldi et al. (2018) reported that procyanidin B2 and epicatechin in avocado peel and trans-5-O-caffeoyl-D-quinic acid, procyanidin $\mathrm{B} 1$, catechin, and epicatechin in avocado seed played a key role in antioxidant and anti-inflammatory activities. It could be concluded that the selected avocado by-products in this study were valuable sources of antioxidants, especially phenolic compounds.

\section{Melanosis evaluation}

Fig. 2 shows the melanosis scores in shrimp carapace 


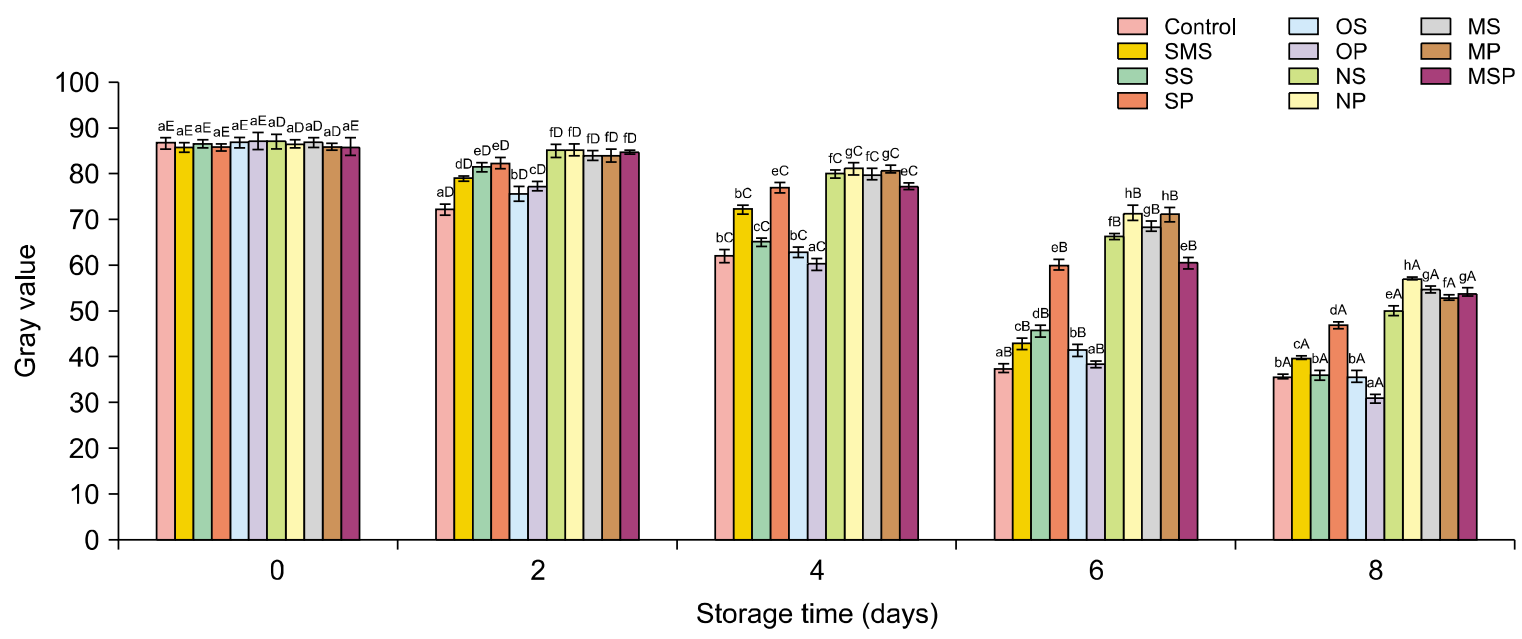

Fig. 2. Changes in mean gray values of the carapace area of whiteleg shrimp, Litopenaus vannamei treated with different extracts [Sap seed (SS), Sap peel (SP), 034 seed (OS), 034 peel (OP), Nuoc seed (NS), Nuoc peel (NP), seed mixture (MS), peel mixture (MP), and peel and seed mixture (MSP)] during refrigerated storage, compared with that of sodium metabisulfite (SMS) and the control. Different uppercase letters (A-D) are related to significant differences among other storage days in the same treatment $(P<0.05)$. Different lowercase letters $(\mathrm{a}-\mathrm{g})$ showed significant differences among other treatments in the same storage time $(P<0.05)$.

treated with avocado by-product extracts $(0.025 \%, \mathrm{w} / \mathrm{v})$, SMS $(1.25 \%, \mathrm{w} / \mathrm{v})$, and the control (with water) during 8 days storage at $4^{\circ} \mathrm{C}$. Changes in mean gray value were the indication of melanosis. It is also worth noting that high melanosis prevention level was associated with low decreasing level in mean gray value during storage. At day 0 , there were no significant differences in the gray values $(P>0.05)$ among the shrimp samples. However, after 4 days storage, most samples treated with the prepared extracts (SP, NS, NP, MS, MP, and MSP) demonstrated a slight decline in the mean gray values, which showed the effective prevention of melanosis. The mean gray values then significantly decreased from day 4 to day 6 but the changes were limited the last 2 days $(P<0.05)$. Consequently, after 8 days of storage, samples treated with the six extracts (NP, NS, SP, MS, MP, and MSP) had mean gray values ranging from $47.0 \pm 0.7$ to $57.3 \pm 0.4 \%$ that were significantly higher than those in SMS-treated samples (the mean gray value of $39.8 \pm 0.4 \%$ ), meaning that the six extracts demonstrated better melanosis prevention than SMS. Among them, NP extract showed the highest efficiency of retarding melanosis in shrimp with the mean gray value of $57.3 \pm 0.4 \%$. Another noticeable point was that samples stored with ternary or multiple mixtures demonstrated higher mean gray values than those stored with single extracts, except for NP $(P<0.05)$. Other plant extracts from oyster mushroom (Llanto and Encarnacion, 2018), pomegranate (Basiri et al., 2015; Yuan et al., 2016a), green tea (Nirmal and Benjakul, 2012; Yuan et al., 2016b), and grape seed (Sun et al., 2014) were also reported to retard melanosis. For instance, whiteleg shrimp treated with Pleurotus ostreatus, mushroom extract recorded a relative change of about $80 \%$ after 3 days storage (Llanto and Encarnacion, 2018), which was comparable to the prepared extracts in this study after 4 days storage at similar treatments with mean gray values in the range of 77.10 to $81.12 \%$ for NP, MS, MSP, MP, NS, and SP. Overall, the results showed the paramount importance of adding value to avocado byproducts for melanosis treatment of whiteleg shrimp as a cheap natural source and substituting for SMS.

\section{Lipid peroxidation inhibition}

Fig. 3 shows changes in TBARS values of whiteleg shrimp treated with the prepared extracts $(0.025 \%$, w/v), SMS $(1.25 \%, \mathrm{w} / \mathrm{v})$, and control (deionized water). No significant difference was observed in TBARS values among all samples at day $0(P>0.05)$. The TBARS values of all treatment batches overall showed an upward trend at various degrees $(P<0.05)$, indicating an increase in lipid peroxidation over storage. It is noticeable that all extracts of avocado by-products impeded the degree of lipid peroxidation compared with SMS and the control groups during 8 days storage $(P<0.05)$. Among the samples, the NP treatment had the smallest TBARS value of $3.3 \pm 0.1$ (mg $\mathrm{MDA} / \mathrm{kg}$ ), whereas the OS treatment showed the highest TBARS value of $5.5 \pm 0.1(\mathrm{mg} \mathrm{MDA} / \mathrm{kg})$ at the end of the period $(P<0.05)$. These findings indicated that extracts of avocado by-products could mitigate the peroxidation of lipids in shrimp samples during storage, which was also reported in the literature for various plant extracts. Basiri et al. (2015) revealed that after 10 days of refrigerated storage, whiteleg shrimp treated with pomegranate peel extract had 30\% lower TBARS values compared to the control group. Similarly, Abbasvali et al. (2016) reported that aqueous saffron (Crocus sativus) tepal extract inhibited lipid oxidation in treated shrimp during 9 days of iced storage. Other authors reported a delay in the TBARS 


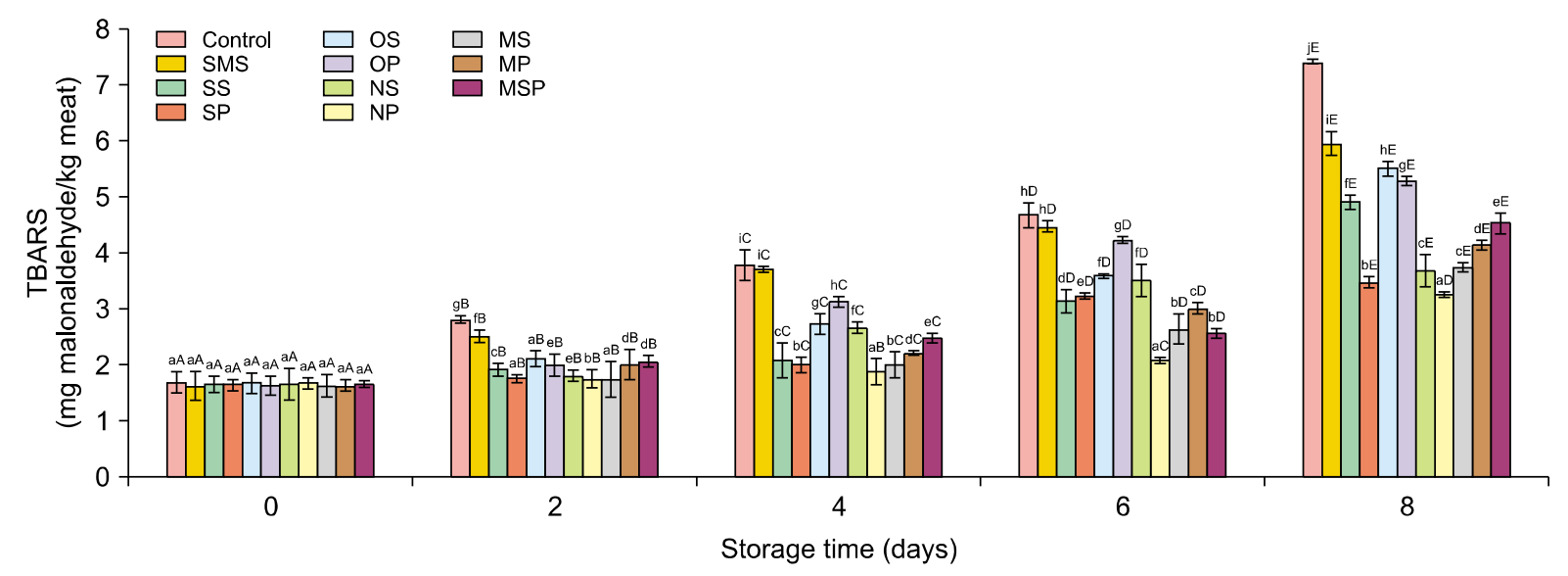

Fig. 3. Changes in TBARS values of whiteleg shrimp, Litopenaus vannamei treated with different extracts [Sap seed (SS), Sap peel (SP), 034 seed (OS), 034 peel (OP), Nuoc seed (NS), Nuoc peel (NP), seed mixture (MS), peel mixture (MP), and peel and seed mixture (MSP)] during refrigerated storage, compared with that of sodium metabisulfite (SMS) and the control. Different uppercase letters $(A-E)$ are related to significant differences among other storage days in the same treatment $(P<0.05)$. Different lowercase letters $(a-h)$ showed significant differences among other treatments in the same storage time $(P<0.05)$.

values in L. vannamei shrimp treated with chamuang (Garcinia cowa Roxb.) leaf extract (Shiekh et al., 2019), a green tea extract in combination with ascorbic acid (Nirmal and Benjakul, 2012), and a coat with quince seed mucilage and green tea extract (Noshad et al., 2017).

\section{Antioxidant activities}

DPPH radical scavenging assay: Fig. 4 shows the result of DPPH radical scavenging activity of the prepared extracts, acting in a dose-dependent manner. It is noted that, for each type of avocado, the peel extract possessed considerably higher activity than the seed extract, which was also observed for the mixtures containing peels. Among the extracts, at the highest concentration of $100 \mu \mathrm{g} / \mathrm{mL}$, SP exhibited the highest inhibitory activity with $97.0 \pm 0.5$ $\%$ DPPH scavenging activity $\left(\mathrm{IC}_{50}=3.6 \mu \mathrm{g} / \mathrm{mL}\right)$, whereas OS showed the lowest activity with $73.4 \pm 1.0 \%$ scaveng- ing activity $\left(\mathrm{IC}_{50}=63.1 \mu \mathrm{g} / \mathrm{mL}\right)$. Notably, no significant difference $(P>0.05)$ was observed in the DPPH scavenging activity among ternary, and multiple mixtures with the inhibition level ranging from $94.3 \pm 0.1 \%$ to $94.9 \pm$ $0.3 \%\left(\mathrm{IC}_{50}=5.5 \sim 8.6 \mu \mathrm{g} / \mathrm{mL}\right)$.

At the highest concentration of $100 \mu \mathrm{g} / \mathrm{mL}$, the prepared extracts displayed DPPH scavenging percentage between $73.4 \pm 1.0$ and $99.0 \pm 0.5 \%$ and $\mathrm{IC}_{50}$ values of 3.6 to $63.1 \mu \mathrm{g} / \mathrm{mL}$. It is noted that the scavenging activity of avocado by-products in this study was significantly higher than those of avocado peels and seeds in previous studies. Melgar et al. (2018) showed that $\mathrm{IC}_{50}$ values calculated from a DPPH radical scavenging assay for the hydroethanolic extracts of Persea america Mill. var. Hass peel and kernel were $149 \pm 5$ and $220 \pm 3 \mu \mathrm{g} / \mathrm{mL}$, respectively. Antasionasti et al. (2017) reported that the most active fraction of methanolic extract from avocado peel was able

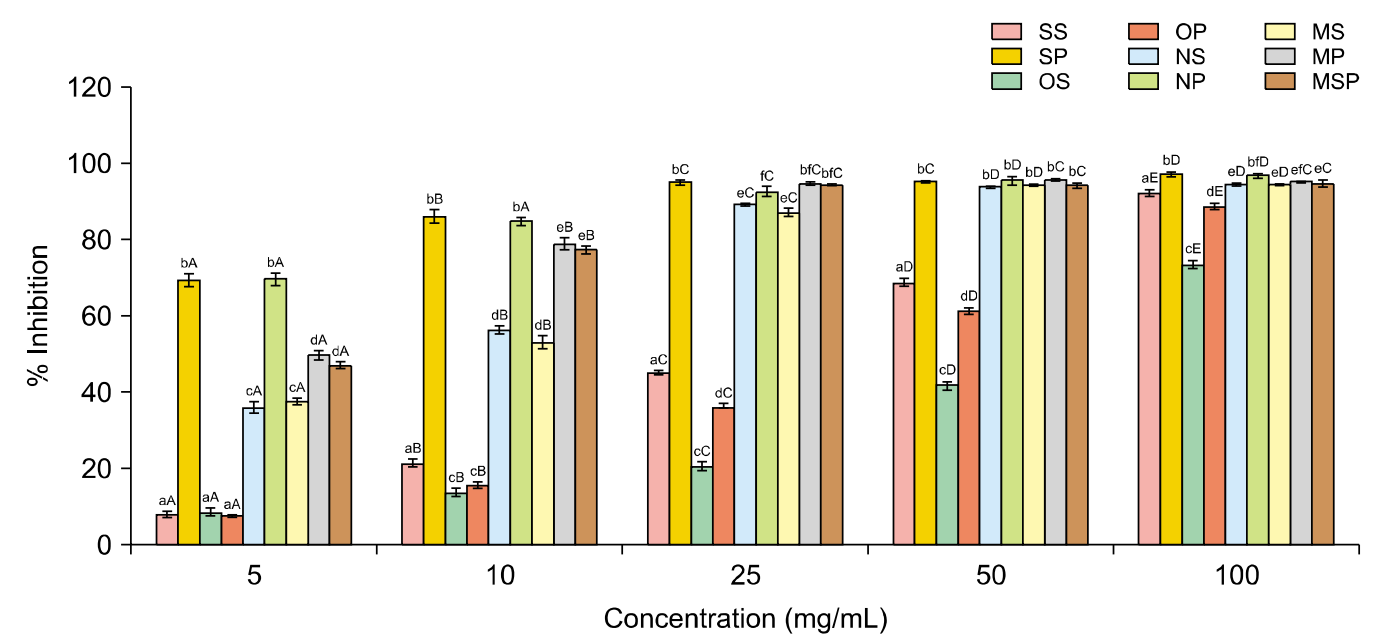

Fig. 4. DPPH radical inhibitory activity of 9 avocado by-product extracts [Sap seed (SS), Sap peel (SP), 034 seed (OS), 034 peel $(\mathrm{OP})$, Nuoc seed (NS), Nuoc peel (NP), seed mixture (MS), peel mixture (MP), and peel and seed mixture (MSP)]. Different uppercase letters $(A-E)$ are related to significant differences among other storage days in the same treatment $(P<0.05)$. Different lowercase letters (a-f) showed significant differences among other treatments in the same storage time $(P<0.05)$. 


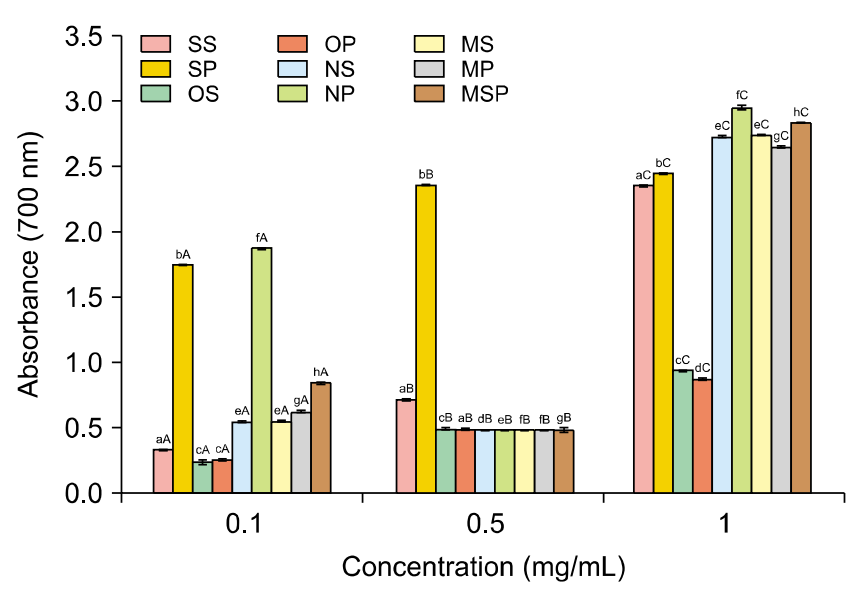

Fig. 5. Reducing power of 9 avocado by-product extracts [Sap seed (SS), Sap peel (SP), 034 seed (OS), 034 peel (OP), Nuoc seed (NS), Nuoc peel (NP), seed mixture (MS), peel mixture (MP), and peel and seed mixture (MSP)]. Different uppercase letters $(A-C)$ are related to significant differences among other storage days in the same treatment $(P<0.05)$. Different lowercase letters (a-f) showed significant differences among other treatments in the same storage time $(P<0.05)$.

to scavenge DPPH with an $\mathrm{IC}_{50}$ value of $4,221 \pm 137 \mu \mathrm{g} /$ $\mathrm{mL}$. The high DPPH scavenging activity of these investigated extracts was consistent with their high TPC (Fig. 2). A possible explanation was that phenolic compounds could provide hydrogen atoms or donate electrons, which quenched DPPH free radicals to stabilize diamagnetic molecules, leading to a decrease in the absorbance (Mathew et al., 2015).

Ferric reducing power assay: The antioxidant activity of the prepared extracts analyzed via FRAP assay was shown in Fig. 5. Higher absorbance indicates stronger reducing power. Like the DPPH scavenging activity, the ferric reducing power of these extracts augmented as their concentrations increased $(P<0.05)$. At the concentration of $1.0 \mathrm{mg} / \mathrm{mL}$, the absorbance of all extracts was in the range of $0.8890 \pm 0.0085$ to $2.9547 \pm 0.0025$. The NP ex- tract possessed the highest FRAP, while the OP extract showed the lowest activity $(P<0.05)$. These ferric reducing capacities could be comparable to those polyphenols and flavonoids, such as callistephin, malvin, ID-8, silychristin, pelargonin, oenin, 2,4,6-trihydroxybenzaldehyde, 3,4-dihydroxy-5-methoxybenzoic acid, and arachidonoyl dopamine (the absorbance range from 0.615 to 2.430 at $30 \mu \mathrm{g} / \mathrm{mL}$ ) (Huyut et al., 2017). It indicates that the byproducts investigated in this study were great antioxidant sources for potential food applications.

Tyrosinase enzyme inhibition assay: Fig. 6 shows the effects of the prepared extracts on the tyrosinase inhibition using L-DOPA as a substrate. Like DPPH and FRAP assays, all the extracts illustrated positive effects on the tyrosinase inhibition with a dose-dependent property. The level of inhibition at the highest concentration of $100 \mu \mathrm{g} / \mathrm{mL}$ ranged from $32.6 \pm 2.1$ to $65.8 \pm 2.8$. Among the nine extracts, those from 'Nuoc' and 'Sap' avocado peels and seeds (i.e., NP, NS, SP, and SS) exhibited better inhibition levels [I>50\%, IC 50 values from 48.0 to $94.6 \mu \mathrm{g} / \mathrm{mL}$ $(P<0.05)]$ as compared to the other five extracts. In addition, tyrosinase inhibition capacities of 'Nuoc' and 'Sap' avocado peel and seed extracts were similar to most of the 50 methanolic extracts from Sudanese medicinal plants whose inhibition levels were from 1.58 to $84.39 \%$ at $125 \mu \mathrm{g} / \mathrm{mL}$ (Muddathir et al., 2017). Laksmiani et al. (2020) found that the tyrosinase inhibitory activity of ethyl acetate extract of avocado seed possessed an $\mathrm{IC}_{50}$ value of $93.02 \mu \mathrm{g} / \mathrm{mL}$, which was lower than that for NP, SP, and NS in this study. This high inhibitory activity towards tyrosinase of the prepared extracts could be attributed to their high content of polyphenols and strong antioxidant activity. It is likely that active antioxidant compounds in these extracts could retard the initial step of radical species creation and reduce the supply of oxygen during the tyrosinase reaction, leading to the inhibition of tyrosinase. Another possible reason is that some active

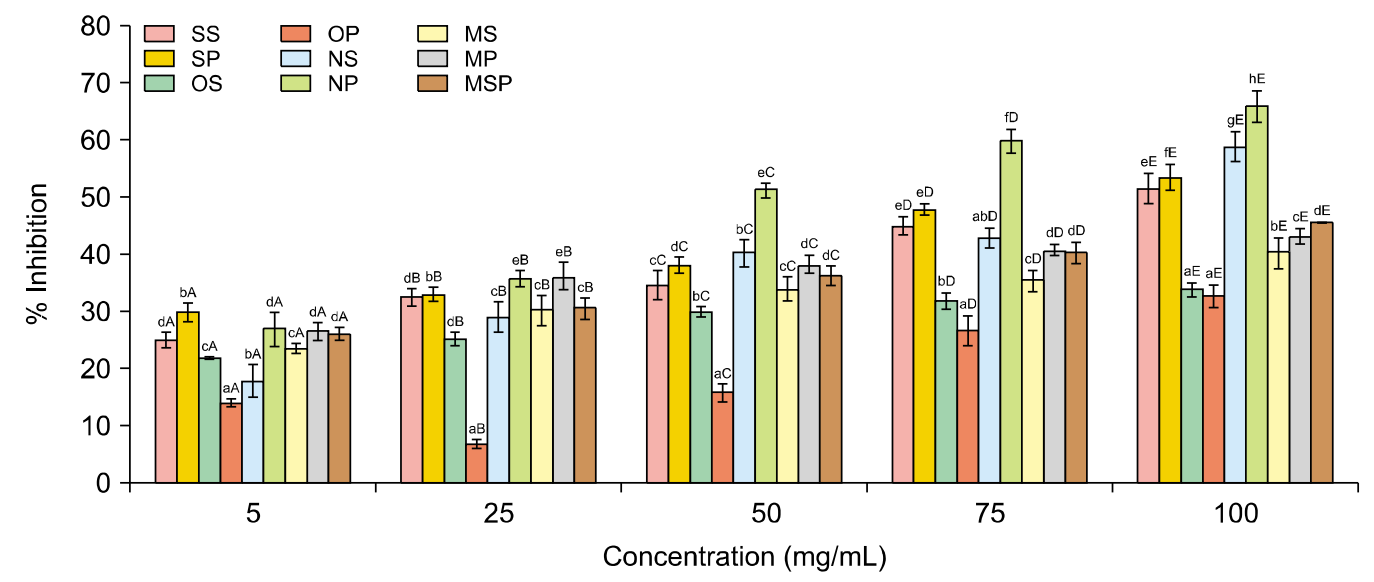

Fig. 6. Tyrosinase inhibitory activity of 9 avocado by-product extracts [Sap seed (SS), Sap peel (SP), 034 seed (OS), 034 peel (OP), Nuoc seed (NS), Nuoc peel (NP), seed mixture (MS), peel mixture (MP), and peel and seed mixture (MSP)]. Different uppercase letters $(A-E)$ are related to significant differences among other storage days in the same treatment $(P<0.05)$. Different lowercase letters (a-h) showed significant differences among other treatments in the same storage time $(P<0.05)$. 
antioxidants in these extracts could prevent the catalytic activity of tyrosinase (Cui et al., 2018).

Melanosis in shrimp was characterized by the formation of black spots caused by the oxidation of phenol substrates (such as tyrosine) to quinones catalyzed by polyphenoloxidase (Gonçalves and de Oliveira, 2016; Pan et al., 2019). Phenolic compounds could alleviate melanosis formation in different mechanisms based on the elimination of one or more important components involved in the enzymatic reaction, such as oxygen, copper, substrate, and the enzyme itself. Certain polyphenols can lower PPO activity by direct interaction with PPO through chelating or forming hydrogen bonding and evenly donating an electron to the intermediate quinones, and hence they could interrupt the oxidation process (Sae-leaw and Benjakul, 2019). Several polyphenols have been documented with intense PPO inhibitory activity such as catechin and its derivatives, quercetin and its derivatives, and coumaric acid (Sae-leaw et al., 2017; Rosero et al., 2019). Among various polyphenolic compounds found in avocado byproducts, 30 of them belonged to organic acids, hydroxycinnamic acids, catechins, free and glycosylated flavonoids, and dimeric and trimeric procyanidins. Catechin, epicatechin, six quercetin derivatives, four dimeric procyanidins, and three trimeric procyanidins were identified in the most active fractions of avocado peel and seeds reported in the literature (Rosero et al., 2019). Catechin and its derivatives exhibited tyrosinase inhibitory activity based on the combined effects of metal chelation and the reduction of quinone (Nirmal and Benjakul, 2012). As shown in Fig. 1 the prepared extracts of avocado byproducts were rich in polyphenols, which could explain the significant retardation of melanosis formation in shrimp during storage.

Fig. 7 plots the correlations of the anti-melanosis of the prepared extracts to their TPC values, the oxidant inhibitory activities and lipid peroxidation (TBARS) values at day 6 storage (confidence level of 95\%). The data at the 6 th day was chosen because it showed the most variation in the melanosis score (Fig. 2). It is clearly seen that the melanosis inhibition on shrimp stored up to 6 days had a good correlation with the TPC and antioxidant activities of the corresponding extracts $(r=0.547 \sim 0.857)$. The high correlation coefficient $r$ of 0.811 for the TPC value could confirm the important role of phenolic compounds in the prepared by-product extracts on retarding melanosis formation in shrimp. It should be noted that the scavenging activity toward DPPH radical behaved in the same manner with TPC value toward melanosis inhibitory activity as the DPPH scavenging activity $(r=0.857)$ was known to be consistent with the TPC value (Sun et al., 2014; Sae-leaw and Benjakul, 2019; Shiekh et al., 2019). A moderate correlation with tyrosinase inhibitory activity $(r=0.588)$ indicates that tyrosinase, a common

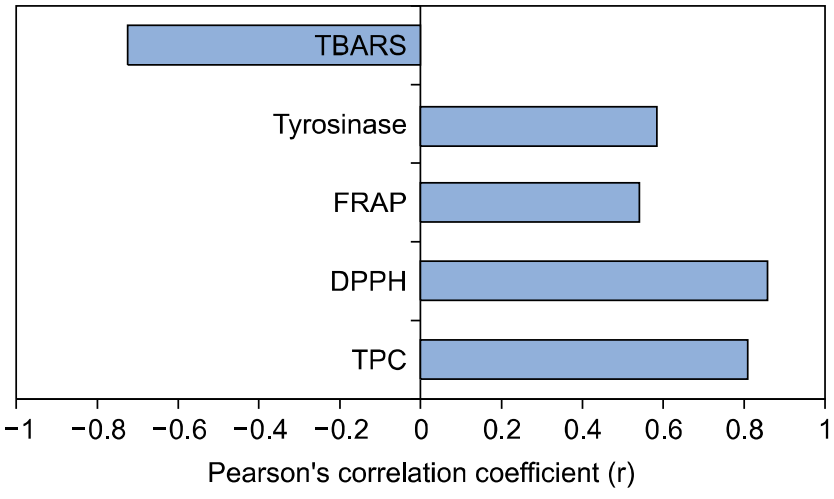

Fig. 7. Pearson's correlation coefficient ( $r$ ) of the melanosis inhibitory activity (at day 6 storage) to total phenolic content (TPC), antioxidant activities (DPPH radical scavenging, ferric reducing power (FRAP) and tyrosinase enzyme inhibition (at concentration of $100 \mu \mathrm{g} / \mathrm{mL}$ ), and TBARS.

enzyme found in crustaceans, also plays a reasonable role in melanosis formation. The lower Pearson coefficient ( $r$ ) for tyrosinase inhibition than TPC value could be explained through different melanosis-inhibition mechanisms during cold storage of shrimp with avocado byproduct extracts. A similar relationship was found for the FRAP data. However, the values for TBARS indicated the decrease in quality of shrimp, which was due to the formation of lipid oxidizing products (e.g., aldehydes produce off flavors) (Chaijan et al., 2006; Zhang et al., 2015) This high absolute $r$ value indicated that retardation of both melanosis and lipid peroxidation were attributed to the high phenolic content in the extracts. Phenolic compounds might chelate the metal pro-oxidants in shrimp muscle and that resulted in the delay of lipid oxidation in shrimp (Abbasvali et al., 2016). Sai-Ut et al. (2020) reported that extract of mango seed kernel possessing rich phenolic compounds could appreciably retard the melanosis formation and lipid oxidation process. Further studies on the influence of phenolic profiles on melanosis prevention and lipid oxidation inhibition should be investigated.

Overall, the present study highlights the promising applications of avocado by-product (seed and peel) extracts as efficient inhibitors of black spots and lipid oxidation during refrigerated storage of whiteleg shrimp. These inhibitory activities varied depending on the type of avocado fruits, which was due to the difference in TPC and antioxidant power among them. Between the investigated samples, the extract of 'Nuoc' avocado peel showed the most promising impact for shrimp preservation with its high TPC (144.7 $\pm 1.9 \mathrm{mg}$ GAE/g DW) and antioxidant activities ( $\mathrm{IC}_{50}$ values for DPPH and PPO inhibition assays of 2.3 and $48.0 \mu \mathrm{g} / \mathrm{mL}$, respectively). These properties were even better than those of the commercial SMS additive at high preserving dose. Therefore, these avocado by-products can be considered as safe and cheap natural 
sources of bioactive compounds for anti-melanosis and extending shelf life of whiteleg shrimp, contributing to environmental pollution prevention and added economic value.

\section{ACKNOWLEDGEMENTS}

This work belongs to the project grant $67 / 2019 / \mathrm{HĐ}$ QPTKHCN funded by Ho Chi Minh City Department of Science and Technology, Viet Nam. The study was supported by Ho Chi Minh City University of Technology and Education, Viet Nam.

\section{AUTHOR DISCLOSURE STATEMENT}

The authors declare no conflict of interest.

\section{REFERENCES}

Abbasvali M, Ranaei A, Shekarforoush SS, Moshtaghi H. The effects of aqueous and alcoholic saffron (Crocus sativus) tepal extracts on quality and shelf-life of pacific white shrimp (Litopeneous vannamei) during iced storage. J Food Qual. 2016. 39: 732-742.

Antasionasti I, Riyanto S, Rohman A. Antioxidant activities and phenolics contents of avocado (Persea americana Mill.) peel in vitro. Res J Med Plants. 2017. 11:55-61.

Azwanida NN. A review on the extraction methods use in medicinal plants, principle, strength and limitation. Med Aromat Plants. 2015. 4:196. https://doi.org/10.4172/2167-0412. 1000196

Basiri S, Shekarforoush SS, Aminlari M, Akbari S. The effect of pomegranate peel extract (PPE) on the polyphenol oxidase (PPO) and quality of Pacific white shrimp (Litopenaeus vannamei) during refrigerated storage. LWT-Food Sci Technol. 2015. 60:1025-1033.

Benjakul S, Bauer F. Biochemical and physicochemical changes in catfish (Silurus glanis Linne) muscle as influenced by different freeze-thaw cycles. Food Chem. 2001. 72:207-217.

Chaijan M, Benjakul S, Visessanguan W, Faustman C. Changes of lipids in sardine (Sardinella gibbosa) muscle during iced storage. Food Chem. 2006. 99:83-91.

Cui HX, Duan FF, Jia SS, Cheng FR, Yuan K. Antioxidant and tyrosinase inhibitory activities of seed oils from Torreya grandis Fort. ex Lindl.. BioMed Res Int. 2018. 2018:5314320. https://doi. org/10.1155/2018/5314320

Djeridane A, Yousfi M, Nadjemi B, Boutassouna D, Stocker P, Vidal N. Antioxidant activity of some Algerian medicinal plants extracts containing phenolic compounds. Food Chem. 2006. 97:654-660.

Encarnacion AB, Fagutao F, Jintasataporn O, Worawattanamateekul W, Hirono I, Ohshima T. Application of ergothioneinerich extract from an edible mushroom Flammulina velutipes for melanosis prevention in shrimp, Penaeus monodon and Litopenaeus vannamei. Food Res Int. 2012. 45:232-237.

European Brewery Convention. Moisture content of malt. 2000 [cited 25 Sep 2019]. Available from: https://brewup.eu/ebcanalytica/malt/moisture-content-of-malt/4.2

Galvão JA, Vázquez-Sánchez D, Yokoyama VA, Savay-Da-Silva
LK, Brazaca SGC, Oetterer M. Effect of 4-hexylresorcinol and sodium metabisulphite on spoilage and melanosis inhibition in Xiphopenaeus kroyeri shrimps. J Food Process Preserv. 2017. 41:e12943. https://doi.org/10.1111/jfpp.12943

Gashahun GS, Solomon LB. Study of the antioxidant activities of avocado (Persea americana Mill.) and three banana (Musa paradisiac L.) varieties by FRAP and Rancimat assays. Anatomy Physiol Biochem Int J: 2018. 6:555678. https://doi.org/10.19080/ APBIJ.2019.06.555678

Githinji P, Gitu L, Marete E, Githua M, Mugo M, Mbaka EN. Quantitative analysis of total phenolic content in avocado (Persia americana) seeds in Eastern province of Kenya. 2013. 3:48-51.

Gómez FS, Sánchez SP, Iradi MGG, Azman NAM, Almajano MP. Avocado seeds: extraction optimization and possible use as antioxidant in food. Antioxidants. 2014. 3:439-454.

Gonçalves AA, de Oliveira ARM. Melanosis in crustaceans: a review. LWT-Food Sci Technol. 2016. 65:791-799.

Huang WY, Zhang HC, Liu WX, Li CY. Survey of antioxidant capacity and phenolic composition of blueberry, blackberry, and strawberry in Nanjing. J Zhejiang Univ Sci B. 2012. 13:94-102.

Huyut Z, Beydemir Ş, Gülçin İ. Antioxidant and antiradical properties of selected flavonoids and phenolic compounds. Biochem Res Int. 2017. 2017:7616791. https://doi.org/10.1155/2017/ 7616791

Jescovitch LN, Ullman C, Rhodes M, Davis DA. Effects of different feed management treatments on water quality for Pacific white shrimp Litopenaeus vannamei. Aquacult Res. 2018. 49:526-531.

Jung HA, Jung YJ, Yoon NY, Jeong DM, Bae HJ, Kim DW, et al. Inhibitory effects of Nelumbo nucifera leaves on rat lens aldose reductase, advanced glycation endproducts formation, and oxidative stress. Food Chem Toxicol. 2008. 46:3818-3826.

Kosińska A, Karamać M, Estrella I, Hernández T, Bartolomé B, Dykes GA. Phenolic compound profiles and antioxidant capacity of Persea americana Mill. peels and seeds of two varieties. J Agric Food Chem. 2012. 60:4613-4619.

Laksmiani NPL, Sanjaya IKN, Leliqia NPE. The activity of avocado (Persea americana Mill.) seed extract containing catechin as a skin lightening agent. J Pharm Pharmacogn Res. 2020. 8:449456.

Llanto MG, Encarnacion AB. Application of edible oyster mushroom, Pleurotus ostreatus extract to control postharvest melanosis in shrimp, Penaeus vannamei. Philipp J Sci. 2018. 147:231-238.

Martínez-Álvarez Ó, Gómez-Guillén MC, Montero P. Chemical and microbial quality indexes of Norwegian lobsters (Nephrops norvegicus) dusted with sulphites. Int J Food Sci Technol. 2008. 43:1099-1110.

Mathew S, Abraham TE, Zakaria ZA. Reactivity of phenolic compounds towards free radicals under in vitro conditions. J Food Sci Technol. 2015. 52:5790-5798.

Melgar B, Dias MI, Ciric A, Sokovic M, Garcia-Castello EM, Rodriguez-Lopez AD, et al. Bioactive characterization of Persea americana Mill. by-products: a rich source of inherent antioxidants. Ind Crops Prod. 2018. 111:212-218.

Molyneux P. The use of the stable free radical diphenylpicrylhydrazyl (DPPH) for estimating antioxidant activity. Songklanakarin J Sci Technol. 2004. 26:211-219.

Muddathir AM, Yamauchi K, Batubara I, Mohieldin EAM, Mitsunaga $T$. Anti-tyrosinase, total phenolic content and antioxidant activity of selected Sudanese medicinal plants. S Afr J Bot. 2017. 109:9-15.

Nirmal NP, Benjakul S. Effect of green tea extract in combination with ascorbic acid on the retardation of melanosis and quality changes of pacific white shrimp during iced storage. Food Bioprocess Technol. 2012. 5:2941-2951.

Nirmal NP, Benjakul S. Inhibition of melanosis formation in Pacific white shrimp by the extract of lead (Leucaena leucocephala) seed. Food Chem. 2011. 128:427-432. 
Noshad M, Nasehi B, Anvar A. Effect of active edible coating made by quince seed mucilage and green tea extract on quality of fried shrimps: physicochemical and sensory properties. Nutr Food Sci Res. 2017. 4:31-36.

Pan C, Chen S, Hao S, Yang X. Effect of low-temperature preservation on quality changes in Pacific white shrimp, Litopenaeus vannamei: a review. J Sci Food Agric. 2019. 99:6121-6128.

Qian Y, Yang S, Ye JX, Xie J. Effect of quercetin-containing preservatives and modified atmospheric packaging on the production of biogenic amines in Pacific white shrimp (Litopenaeus vannamei). Aquacult Fish. 2018. 3:254-259.

Rodríguez-Carpena JG, Morcuende D, Estévez M. Avocado byproducts as inhibitors of color deterioration and lipid and protein oxidation in raw porcine patties subjected to chilled storage. Meat Sci. 2011. 89:166-173.

Rosero JC, Cruz S, Osorio C, Hurtado N. Analysis of phenolic composition of byproducts (seeds and peels) of avocado (Persea americana Mill.) cultivated in Colombia. Molecules. 2019. 24: 3209. https://doi.org/10.3390/molecules24173209

Saavedra J, Córdova A, Navarro R, Díaz-Calderón P, Fuentealba C, Astudillo-Castro C, et al. Industrial avocado waste: functional compounds preservation by convective drying process. J Food Eng. 2017. 198:81-90.

Sae-leaw T, Benjakul S, Simpson BK. Effect of catechin and its derivatives on inhibition of polyphenoloxidase and melanosis of Pacific white shrimp. J Food Sci Technol. 2017. 54:1098-1107.

Sae-leaw T, Benjakul S. Prevention of melanosis in crustaceans by plant polyphenols: a review. Trends Food Sci Technol. 2019. 85:1-9.

Sai-Ut S, Noknoi N, Nakjai N. Effect of mango seed kernel extract on polyphenol oxidase inhibition and shelf life of shrimp dur- ing iced storage. Agric Nat Resour. 2020. 54:641-648.

Shiekh KA, Benjakul S, Sae-leaw T. Effect of Chamuang (Garcinia cowa Roxb.) leaf extract on inhibition of melanosis and quality changes of Pacific white shrimp during refrigerated storage. Food Chem. 2019. 270:554-561.

Sun H, Lv H, Yuan G, Fang X. Effect of grape seed extracts on the melanosis and quality of Pacific white shrimp (Litopenaeus vannamei) during iced storage. Food Sci Technol Res. 2014. 20:671677.

Tremocoldi MA, Rosalen PL, Franchin M, Massarioli AP, Denny C, Daiuto ÉR, et al. Exploration of avocado by-products as natural sources of bioactive compounds. PLoS One. 2018. 13: e0192577. https://doi.org/10.1371/journal.pone.0192577

Wang W, Bostic TR, Gu L. Antioxidant capacities, procyanidins and pigments in avocados of different strains and cultivars. Food Chem. 2010. 122:1193-1198.

Yen GC, Chen HY. Antioxidant activity of various tea extracts in relation to their antimutagenicity. J Agric Food Chem. 1995. 43:27-32.

Yuan G, Lv H, Tang W, Zhang X, Sun H. Effect of chitosan coating combined with pomegranate peel extract on the quality of $\mathrm{Pa}$ cific white shrimp during iced storage. Food Control. 2016a. 59:818-823.

Yuan G, Zhang X, Tang W, Sun H. Effect of chitosan coating combined with green tea extract on the melanosis and quality of $\mathrm{Pa}$ cific white shrimp during storage in ice. CyTA-J Food. 2016b. 14:35-40.

Zhang B, Ma LK, Deng SG, Xie C, Qiu XH. Shelf-life of pacific white shrimp (Litopenaeus vannamei) as affected by weakly acidic electrolyzed water ice-glazing and modified atmosphere packaging. Food Control. 2015. 51:114-121. 